\title{
Valuation of a Basket Loan Credit Default Swap
}

\author{
Jin Liang (Corresponding author) \\ Department of Mathematics, Tongji University, \\ Shanghai 200092, P.R.China \\ Tel: +86-21-6598-3240 ext. 206 E-mail: liang_jin@tongji.edu.cn
}

Yujing Zhou

Department of Mathematics, Tongji University, Shanghai 200092, P.R.China

Tel: +86-21-6598-3240 ext. 206 E-mail: nayi_crystal@126.com

Received: September 16, $2010 \quad$ Accepted: September 29, $2010 \quad$ doi:10.5430/ijfr.v1n1p21

This work is supported by National Basic Research Program of China (973 Program) 2007CB814903

\begin{abstract}
This paper provides a methodology for valuing a basket Loan CDS (LCDS) by considering both default and prepayment risks. Under "top down" and intensity framework, using a single-factor model, correlated default and prepayment risks are considered, where the stochastic interest rate is used to be their common factor. All stochastic processes in the model are assumed to follow CIR processes. Through Feynman-Kac formula, we obtain a PDE problem and its closed-form solution. Numerical examples are provided.
\end{abstract}

Keywords: LCDS, Default, Prepayment, PDE method, Closed-form solution

\section{Introduction}

Loan-only Credit Default Swaps, called LCDS in simple, are financial instruments that provide the buyer an insurance against the default of the underlying syndicated secured loan. Its markets were launched in 2006 both in US and Europe. It is developed on the basis of standard Credit Default Swaps (CDS). A standard Credit Default Swap is a kind of insurance against credit risk. The buyer of the CDS is the buyer of protection who pays a fixed fee or premium to the seller of protection for a period of time. If the credit event occurs, the seller pays compensation to the buyer. If there is no credit event occurs during the term of swap, the buyer continues to pay the premium until the CDS maturity. Hull-White (Hull and White, 2000) first considered the valuation of a standard credit default swap when there is no counterparty default risk. And they extended their study to the situation where there is possibility of counterparty default risk and obtaining a pricing formula with Monte Carlo simulation (Hull and White, 2001). Furthermore, they develop two fast procedures for valuing tranches of collateralized debt obligations and $n^{\text {th }}$ to default CDS without Monte Carlo simulation. (Hull and White, 2004)

Comparing to a standard CDS, a LCDS contract is almost the same, except that 1 . Its reference obligation is limited on loans; 2. It can be cancelled. So that, the pricing of LCDS must take into account not only default probabilities with recovery rate, but also the prepayment probabilities. These two probabilities are negative correlated. The stronger the relationship between default and prepayment is, the higher the LCDS spread will be.

In the literature, different models for LCDS have been developed. These models can be classified into two main categories known as structural models and reduced form models. In these models, default and prepayment are modeled as a function of a set of state variables. The intensity based model is reduced form one. For the pricing LCDS, Zhen Wei (Zhen Wei, 2007) considered a single name LCDS, where default intensity $\lambda_{d}$ and prepayment intensity $\lambda_{p}$ were involved. He used a single-factor model with common factor $X$ and correlation coefficient $\rho$ to depict the negative relationship between default and prepayment. Through a double stochastic process and under CIR process, he solved the 
problem. Based on Zhen Wei's work, Peter Dobranszky (Dobranszky, 2008) times the prepayment intensity $\lambda_{p}$ with a coefficient variation to describe the relationship between LCDS and CDS.

For pricing a basket LCDS, the reduced form model is used more frequently due to the scale and complexity of the pool. The reduced form model can be classified to two categories: "bottom up" and "top down". In a bottom up model, the portfolio intensity is an aggregate of the constituent intensities. In a top down model, the collateral portfolio is modeled as a whole, instead of drilling down to individual constituents; the portfolio intensity is specified without reference to the constituents. The constituent intensities are recovered by random thinning. The benefit of a top-down approach is its simplicity as a result of not having to model the individual constituents of the underlying portfolio. Kay Giesecke (Giesecke, 2008) contrasts these two modeling approaches. It emphasized the role of the information filtration as a modeling tool. S.Wu, LS.Jiang and J.Liang (Jiang and Liang, 2008) use top-down model to pricing of MBS with repayment risk. Using bottom up framework, Shek, H., S. Uematsu and Zhen Wei (Uematsu and Wei, 2007) studied pricing a CDS referenced a pool loan, described the default and prepayment by single-factor Gaussian Copula model. They obtained the spread of the LCDX through Monte Carlo simulation. Dobranszky and Schoutens (Dobranszky and Schoutens, 2007) used single-name Lévy copula to describe the relationship between default and prepayment.

In this study, under "top down" framework, we consider the valuation of a LCDS, where the references pool is considered as an entity. Thus, in this model, the default and the prepayment here become "loss" of the pool by different way, while the inverse prepayment behaviors as "gain". In the following section, we introduce two processes of default and prepayment, and construct the model of LCDS in the continuous time situation. In the third section, we use single-factor model to describe the relationship between default and prepayment through interest rate and other CIR processes. Moreover, we develop a two dimensional partial differential equation. Under the assumption that the PDE has radiation structure solution, the equation can be separated into one ODE problems and one Riccati equation, which can obtain a closed-form of the spread. In the fourth section, numerical examples are shown.

\section{Modeling LCDS}

As mentioned before, the main point of LCDS is a probability that the loan prepays earlier and hence the instrument is cancellable. During the life of an LCDS contract, two kinds of events may be triggered, either the underlying loan is prepaid or the loan-taker goes to default. If a prepayment event were triggered first, the LCDS would have been cancelled. If during the life of the LCDS contract a default event were triggered first, the LCDS would have defaulted, when the LCDS issuer would have to pay the recovery adjusted notional amount to the LCDS buyer.

We consider a basket of loans, from the idea of "top-down". We denote by $A_{t}$ the outstanding principal balance at time $t$. Without loss of generality, we assume that the outstanding balance in pool at time $t=0$ is equal to 1 , that is $A_{0}=1$. Any default event or prepayment event will affect, in general reduce, the total principal in the pool. We denote by $D_{t}$ as the accumulative amount of default and $P_{t}$ as the accumulative amount of prepayment which result in the decrease of total principal at time $t$. Then for any $t>0, A_{t}+D_{t}+P_{t}=1$.

In our model, a continuous time model is considered. We model default and prepayment by introducing stochastic processes $\left(\lambda_{i}^{d}\right)_{t>0}$ and $\left(\lambda_{t}^{p}\right)_{t>0}$. $\lambda_{i}^{d}$ and $\lambda_{i}^{p}$ are the fraction of accumulative default and prepayment amounts over the outstanding balance in a unit time at time $t$ respectively. $\lambda_{t}^{d}$ and $\lambda_{t}^{p}$ are called intensity default and prepayment rates respectively. $\lambda_{t}^{d} \geq 0$, while the sign of $\lambda_{t}^{p}$ is uncertain. If $\lambda_{t}^{{ }^{p} \geq 0}$, it is at prepayment state, otherwise it is at inverse prepayment or complementary lending state. Therefore, for all time ${ }^{t}$, the accumulative default amount ${ }^{D_{t}}$, and outstanding balance $A_{t}$ are non-negative while accumulative prepayment amount $P_{t}$ may negative some time. $D_{t}$ is increase, $A_{t}$ is decrease and $P_{t}$ may be increase or decrease.

At time ${ }^{t}$, in a small time interval $(s, s+d s),(s>t)$, the changes of accumulative amount of default and prepayment are $d D_{s} \square D_{s+d s}-D_{s}$ and $d P_{s} \square P_{s+d s}-P_{s}$ respectively. According to the definitions of $\lambda_{s}^{d}$ and ${ }^{\lambda_{s}^{p}}$, we have

$$
\lambda_{s}^{d}=\frac{d D_{s}}{A_{s} d s}
$$

and

$$
\lambda_{s}^{p}=\frac{d P_{s}}{A_{s} d s}
$$

Plus the equation (1) and (2)

$$
d D_{s}+d P_{s}=A_{s}\left(\lambda_{s}^{d}+\lambda_{s}^{p}\right) d s
$$


It is noticed here that 1 . The meaning of $\lambda_{t}^{d}$ and $\lambda_{t}^{p}$ is different from the ones in reference (Zhen Wei, 2007); 2 . The assumption of the negative possibility of $\lambda_{t}^{p}$ is only available for the basket portfolio, where the top down model is applied. For the single name one, the negative $\lambda_{t}^{p}$ does not make sense.

We denote $A_{t}^{0}$ as the outstanding balance at time $t$ which has been known. Because of $A_{s}+D_{s}+P_{s}=1$, then $A_{s}$ satisfies the following boundary problem of a differential equation:

$$
\left\{\begin{array}{l}
\frac{d A_{s}}{A_{s}}=-\left(\lambda_{s}^{d}+\lambda_{s}^{p}\right) d s, \\
A_{t}=A_{t}^{0} .
\end{array}\right.
$$

Solving the equation (3) gives the following expressions for $A_{s}$

$$
A_{s}=A_{t}^{0} e^{-\int_{t}^{s}\left(\lambda_{u}^{d}+\lambda_{u}^{p}\right) d u} .
$$

It is not difficult to see that once $\lambda_{s}^{d}$ and $\lambda_{s}^{p}$ are explained, $A_{s}$ can be obtained.

Now suppose that the spread rate of LCDS for protection is denoted with c. If the protection fees of the LCDS are payable continuously, then it results that the present value of the fee leg at time ${ }^{t}$ equals the expected value of all fees paid.

$$
P V_{\text {Fee }}=c E_{t}\left[\int_{t}^{T} A_{\tau} B(t, \tau) d \tau\right]
$$

where ${ }^{B(t, \tau)}$ denotes the risk-free discount factor from time $\tau$ to time $t$.

The protection leg, the expected present value of the losses in case of default equals

$$
P V_{\text {Loss }}=E_{t}\left[\int_{t}^{T}(1-R) d D_{\tau} B(t, \tau)\right],
$$

where $R$ is the recovery rate, which assumed to be a positive constant smaller than 1 .

Under the condition of risk neutral, if the contract starts at time $t$, where there is no entry fee, i.e. $P V_{F e e}=P V_{\text {Loss }}$. Thus we get

$$
c=\frac{E_{t}\left[\int_{t}^{T}(1-R) B(t, \tau) d D_{\tau}\right]}{E_{t}\left[\int_{t}^{T} A_{\tau} B(t, \tau) d \tau\right]} .
$$

Let $r_{t}$ be the risk-free interest rate, the discount factor $B(t, \tau)=e^{-\int_{t}^{\tau} r^{t} d u}$. Therefore by (1),

$$
d D_{\tau}=\lambda_{\tau}^{d} A_{\tau} d \tau=A_{t}^{0} \lambda_{\tau}^{d} e^{-\int_{t}^{\tau} \lambda_{u}^{d}+\lambda_{u}^{p} d u} d \tau .
$$

Taking equation (4) and equation (6) into equation (5), we get the spread is

$$
c=\frac{E_{t}\left[\int_{t}^{T}(1-R) A_{t}^{0} \lambda_{\tau}^{d} e^{-\int_{t}^{T}\left(\lambda_{u}^{d}+\lambda_{u}^{p}+r_{u}\right) d u} d \tau\right]}{E_{t}\left[\int_{t}^{T} A_{t}^{0} e^{-\int_{t}^{\tau}\left(\lambda_{u}^{d}+\lambda_{u}^{p}+r_{u}\right) d u} d \tau\right]}=\frac{(1-R) \int_{t}^{T} E_{t}\left[\lambda_{\tau}^{d} e^{-\int_{t}^{\tau}\left(\lambda_{u}^{d}+\lambda_{u}^{p}+r_{u}\right) d u}\right] d \tau}{\int_{t}^{T} E_{t}\left[e^{-\int_{t}^{\tau}\left(\lambda_{u}^{d}+\lambda_{u}^{p}+r_{u}\right) d u}\right] d \tau} .
$$

This is the pricing formula. Now the problem turns to find those expectations.

\section{The solution of LCDS with single-factor model}

\subsection{The correlation between default and prepayment}

The default and prepayment rates are negative correlated. The more relevant is, the higher spread rate will be. Now we use single-factor model to describe the relationship between default rate and prepayment rate. That is

$$
\lambda_{t}^{d}=X_{t}^{0}+X_{t}^{d} \quad \lambda_{t}^{p}=\rho X_{t}^{0}+X_{t}^{p}
$$

where $X_{t}^{0}$ is a common factor of $\lambda_{t}^{d}$ and $\lambda_{t}^{p} ;-1<\rho<0$ is the correlation coefficient which describe the negative correlation between $\lambda_{t}^{d}$ and $\lambda_{t}^{p} ; X_{t}^{d}, X_{t}^{p}$ are the specific factor of $\lambda_{t}^{d}$ and $\lambda_{t}^{p}$ respectively. $X_{t}^{0}, X_{t}^{d}$ and $X_{t}^{p}$ are 
three independent processes.

The motivation and factors that affect the prepayment are complex. Researchers have shown that the prepayment of loans is affected by interest rate, macroeconomic factors, and seasonal factors and so on. Among them, the interest rate is the most important one. When interest rate decreases, borrowers refinance their loans, which led to higher prepayment rate. This means that when the current mortgage interest rate below the contract rate to a certain extent, the borrowers can take loans from other banks to repay the existing loans. As long as the spread reached a certain level, and covered the transaction costs sufficiently, the borrower will have a strong incentive to refinance.

But there is an opposite situation for default events. The cost of borrowing increases with the interest rate increasing, which increases the likelihood of default. Also default has close relation with the borrower's financial condition, macroeconomic and so on.

Measuring these factors, it is suitable to use the interest rate as the common factor of default rate and prepayment rate. So we take ${ }^{X_{t}^{0}=\eta r_{t}}$, for some ${ }^{\eta>0}$. Thus we have

$$
\begin{aligned}
& \lambda_{t}^{d}=\eta r_{t}+X_{t}^{d} \\
& \lambda_{t}^{p}=\eta \rho r_{t}+X_{t}^{p}
\end{aligned}
$$

If $r_{t}$ and $X_{t}^{d}$ are positive processes, the $\lambda_{t}^{d}$ will be a nonnegative process, but $\lambda_{t}^{p}$ may be negative some time as we mentioned before.

\subsection{The solution under CIR process}

Now, we give a model to describe the behavior of ${ }^{r_{t}}, X_{t}^{d}, X_{t}^{p}$. We assume that ${ }^{r_{t}}, X_{t}^{d}, X_{t}^{p}$ follows the stochastic process of CIR, i.e.

$$
\begin{gathered}
d r_{t}=\kappa\left(\theta-r_{t}\right) d t+\sigma \sqrt{r_{t}} d W_{t}, \\
d X_{t}^{i}=\kappa^{i}\left(\theta^{i}-X_{t}^{i}\right) d t+\sigma^{i} \sqrt{X_{t}^{i}} d W_{t}^{i}, \quad i=d, p,
\end{gathered}
$$

where $\kappa, \theta, \sigma, \kappa^{i}, \theta^{i}, \sigma^{i}$ are all positive constants; ${ }^{W_{t}}, W_{t}^{i}$ follow standard Brownian motion. Under this assumption, ${ }^{r_{t}}$, $X_{t}^{i}$ are mean reverting processes. The parameters $\theta, \theta^{i}$ are the "long-run-mean"; $\kappa, \kappa^{i}$ are the mean-reversion rate; $\sigma, \sigma^{i}$ is the volatility. It is well know that, under the condition $2 \kappa \theta>\sigma^{2}, 2 \kappa^{i} \theta^{i}>\left(\sigma^{i}\right)^{i}, r_{t}$ and $X_{t}^{i}$ are nonnegative and their boundary at origin are unattainable.(ex. see Linetsky,2004)

Taking equation (8) and equation (9) into equation (7) and considering the independence among ${ }_{t}^{r_{t}}, X_{t}^{d}$ and $X_{t}^{p}$. The pricing formula (6) changes to

$$
\begin{aligned}
c_{t}= & \frac{(1-R) \int_{t}^{T} E_{t}\left[e^{-\int_{t}^{\tau}\left(\lambda_{u}^{d}+\lambda_{u}^{p}+r_{u}\right) d u} \lambda_{\tau}^{d}\right] d \tau}{\int_{t}^{T} E_{t}\left[e^{-\int_{t}^{T}\left(\lambda_{u}^{d}+\lambda_{u}^{p}+r_{u}\right) d u}\right] d \tau} \\
= & \frac{(1-R) \int_{t}^{T} E_{t}\left[\eta r_{\tau} e^{-(1+\eta(1+\rho)) \int_{t}^{\tau} r_{u} d u}\right] E_{t}\left[e^{-\int_{t}^{\tau} X_{u}^{d} d u}\right] E_{t}\left[e^{-\int_{t}^{\tau} X_{u}^{p} d u}\right] d \tau}{\int_{t}^{T} E_{t}\left[e^{-(1+\eta(1+\rho)) \int_{t}^{\tau} r_{u} d u}\right] E_{t}\left[e^{-\int_{t}^{\tau} X_{u}^{d} d u}\right] E_{t}\left[e^{-\int_{t}^{\tau} X_{u}^{p} d u}\right] d \tau} \\
& +\frac{\int_{t}^{T} E_{t}\left[e^{-(1+\eta(1+\rho)) \int_{t}^{\tau} r_{u} d u}\right] E_{t}\left[X_{\tau}^{d} e^{-\int_{t}^{\tau} X_{u}^{d} d u}\right] E_{t}\left[e^{-\int_{t}^{\tau} X_{u}^{p} d u}\right] d \tau}{\int_{t}^{T} E_{t}\left[e^{-(1+\eta(1+\rho)) \int_{t}^{\tau} r_{t} d u}\right] E_{t}\left[e^{-\int_{t}^{T} X_{u}^{d} d u}\right] E_{t}\left[e^{-\int_{t}^{\tau} X_{u}^{p} d u}\right] d \tau} .
\end{aligned}
$$

\subsection{Solving pricing equation}

$r_{t}, X_{t}^{d}$ and ${ }^{X_{t}^{p}}$ are all CRI processes, in order to calculate the spread, we have to solve two expectations. One 


$$
\begin{gathered}
E_{t}\left[X_{\tau} e^{-a \int_{t}^{\tau} X_{t} d u}\right] \text {, the other is } E_{t}\left[e^{-a \int_{t}^{\tau} X_{t} d u}\right] \text {, where } a \text { is constant and }\left(X_{t}\right)_{t \geq 0} \text { follows the CIR process, satisfied } \\
\qquad d X_{t}=\kappa\left(\theta-X_{t}\right) d t+\sigma \sqrt{X_{t}} d W_{t} .
\end{gathered}
$$

The expectation in equation (10) can be expressed as these two expectations, for example,

$$
\begin{gathered}
E_{t}\left[e^{-(1+\eta(1+\rho)) \int_{t}^{\tau} r_{u} d u}\right]=\left.E_{t}\left[e^{-a \int_{t}^{\tau} X_{u} d u}\right]\right|_{a=1+\eta(1+\rho), r_{t}=X_{t}}, \\
E_{t}\left[r_{\tau} e^{-(1+\eta(1+\rho)) \int_{t}^{\tau} r_{u} d u}\right]=\left.E_{t}\left[r_{\tau} e^{-a \int_{t}^{\tau} X_{u} d u}\right]\right|_{a=1+\eta(1+\rho), r_{t}=X_{t}} .
\end{gathered}
$$

If we can value these two expectations, the pricing equation (10) will be solved.

Now let us solve the two expectations $E_{t}\left[X_{\tau} e^{-a \int_{t}^{\tau} X_{u} d u}\right]$ and $E_{t}\left[e^{-a \int_{t}^{\tau} X_{u} d u}\right]$. Define

$$
I_{t, \tau}^{X}(a, z)=E_{t}\left[e^{-a \int_{t}^{\tau} X_{u} d u+z X_{t}}\right]
$$

then

$$
E_{t}\left[X_{\tau} e^{-a \int_{t}^{\tau} X_{u} d u}\right]=\left.\partial_{z} I_{t, \tau}^{X}(a, z)\right|_{z=0}
$$

and

$$
E_{t}\left[e^{-a \int_{t}^{\tau} X_{u} d u}\right]=\left.I_{t, \tau}^{X}(a, z)\right|_{z=0} .
$$

Now the problem turns to solve $I_{t, \tau}^{X}(a, z)$.

Let $u(x, t)=I_{t, \tau}^{X}(a, z)=E_{t}\left[e^{-a \int_{t}^{\tau} X_{u} d u+z X_{\tau}}\right]$, using the Feynman-Kac formula (e.x. Jiang, 2008), it can be obtained that in the area $\{0 \leq t \leq \tau, 0 \leq X<+\infty\}, u(x, t)$ satisfies the PDE problem

$$
\left\{\begin{array}{l}
u_{t}+\kappa(\theta-x) u_{x}+\frac{1}{2} \sigma^{2} x u_{x x}-a x u=0 \\
u(x, \tau)=e^{z x}
\end{array}\right.
$$

Suppose that this problem has affine structure solution $u(x, t)=e^{A(t)+x B(t)}$, taking it into the equation (14), obtaining that $A(t)$ and $B(t)$ satisfy the following ODE system (15), (16):

$$
\begin{gathered}
\left\{\begin{array}{c}
A^{\prime}(t)+\kappa \theta B(t)=0, \\
A(\tau)=0,
\end{array}\right. \\
\left\{\begin{array}{c}
B^{\prime}(t)-\kappa B(t)+\frac{\sigma^{2}}{2} B^{2}(t)-a=0, \\
B(\tau)=z,
\end{array}\right.
\end{gathered}
$$

We can solve ${ }^{B(t)}$ since equation (16) is a Riccati equation, and $A(t)$ can be easily got once ${ }^{B(t)}$ obtained. Through calculation, we have 


$$
\begin{gathered}
A(t)=-\frac{2 \kappa \theta}{\sigma^{2}} \operatorname{In}\left[\frac{\left(\sigma^{2} z-2 d_{2}\right) e^{-d_{1}(\tau-t)}+\left(2 d_{1}-\sigma^{2} z\right) e^{-d_{2}(\tau-t)}}{\left(\sigma^{2} z-2 d_{2}\right)+\left(2 d_{1}-\sigma^{2} z\right)}\right], \\
B(t)=\frac{2}{\sigma^{2}} \frac{\left(z \sigma^{2}-2 d_{2}\right) d_{1} e^{-d_{1}(\tau-t)}+\left(2 d_{1}-z \sigma^{2}\right) d_{2} e^{-d_{2}(\tau-t)}}{\left(z \sigma^{2}-2 d_{2}\right) e^{-d_{1}(\tau-t)}+\left(2 d_{1}-z \sigma^{2}\right) e^{-d_{2}(\tau-t)}},
\end{gathered}
$$

where $d_{1}=\frac{\kappa-\sqrt{\kappa^{2}+2 a \sigma^{2}}}{2}$ and $d_{2}=\frac{\kappa+\sqrt{\kappa^{2}+2 a \sigma^{2}}}{2}$.

Thus the solution of equation (11) is

$$
\begin{aligned}
I_{t, \tau}^{X}(a, z)= & e^{A(t)+x B(t)} \\
= & {\left[\frac{\left(\sigma^{2} z-2 d_{2}\right) e^{-d_{1}(\tau-t)}+\left(2 d_{1}-\sigma^{2} z\right) e^{-d_{2}(\tau-t)}}{\left(\sigma^{2} z-2 d_{2}\right)+\left(2 d_{1}-\sigma^{2} z\right)}\right]^{-\frac{2 \kappa \theta}{\sigma^{2}}} } \\
& \square e^{\frac{2 x}{\sigma^{2}} \cdot \frac{\left(\sigma^{2} z-2 d_{2}\right) d_{1} e^{-d_{1}(\tau-t)}+\left(2 d_{1}-\sigma^{2} z\right) d_{2} e^{-d_{2}(\tau-t)}}{\left(\sigma^{2} z-2 d_{2}\right) e^{-d_{1}(\tau-t)}+\left(2 d_{1}-\sigma^{2} z\right) e^{-d_{2}(\tau-t)}}}
\end{aligned}
$$

where $d_{1}=\frac{\kappa-\sqrt{\kappa^{2}+2 a \sigma^{2}}}{2}$ and $d_{2}=\frac{\kappa+\sqrt{\kappa^{2}+2 a \sigma^{2}}}{2}$.

Therefore

$$
\begin{aligned}
E_{t}\left[X_{\tau} e^{-a \int_{t}^{\tau} X_{u} d u}\right]= & \left.\partial_{z} I_{t, \tau}^{X}(a, z)\right|_{z=0} \\
= & \left(\kappa \theta \frac{e^{-d_{1}(\tau-t)}-e^{-d_{2}(\tau-t)}}{d_{2} e^{-d_{1}(\tau-t)}-d_{1} e^{-d_{2}(\tau-t)}}+x \frac{\left(d_{1}-d_{2}\right)^{2} e^{-\kappa(\tau-t)}}{\left(-d_{2} e^{-d_{1}(\tau-t)}+d_{1} e^{-d_{2}(\tau-t)}\right)^{2}}\right) \\
& \left\{\frac{d_{2} e^{-d_{1}(\tau-t)}-d_{1} e^{-d_{2}(\tau-t)}}{d_{2}-d_{1}}\right)^{-\frac{2 \kappa \theta}{\sigma^{2}}} \cdot e^{\frac{2 x}{\sigma^{2}} \cdot \frac{d_{1} d_{2}\left(e^{-d_{1}(\tau-t)}-e^{-d_{2}(\tau-t)}\right)}{d_{2} e^{-d_{1}(\tau-t)}-d_{1} e^{-d_{2}(\tau-t)}}} .
\end{aligned}
$$

Also,

$$
\begin{aligned}
E_{t}\left[e^{-a \int_{t}^{\tau} X_{u} d u}\right] & =\left.I_{t, \tau}^{X}(a, z)\right|_{z=0} \\
& =\left(\frac{d_{2} e^{-d_{1}(\tau-t)}-d_{1} e^{-d_{2}(\tau-t)}}{d_{2}-d_{1}}\right)^{-\frac{2 \kappa \theta}{\sigma^{2}}} \cdot e^{\frac{2 x}{\sigma^{2}} \cdot \frac{d_{1} d_{2}\left(e^{-d_{1}(\tau-t)}-e^{-d_{2}(\tau-t)}\right)}{d_{2} e^{-d_{1}(\tau-t)}-d_{1} e^{-d_{2}(\tau-t)}}},
\end{aligned}
$$

where $d_{1}=\frac{\kappa-\sqrt{\kappa^{2}+2 a \sigma^{2}}}{2}$ and $d_{2}=\frac{\kappa+\sqrt{\kappa^{2}+2 a \sigma^{2}}}{2}$.

\subsection{Numerical Examples}

So far, we have derived the solution of the pricing equation (10). Here, we give some examples under the single-factor model.

Now, we take $t=0, R=0.3$. Figures 1-3 show the shape of the LCDS Spread under different parameter sets. The parameter sets and their purposes are summarized in the table 1, the "." in table represents this parameter is variable.

$<$ Table 1 about here $>$

Figure 1 show the shape of LCDS term structures when we fix the common factor $r_{t}, X_{t}^{p}$, which is the specific factor of $\lambda_{t}^{p}$, the correlation parameter $\rho=-0.5$, and vary the mean $\theta^{d}$ and initial value $X_{0}^{d}$ for $X_{t}^{d}$ which is the specific factor of $\lambda_{t}^{d}$. We can see that the LCDS Spread of both figures increases as $\theta^{d}$ or $X_{0}^{d}$ increases. Suppose the other parameters are fixed. If the initial value or mean of specific factor of $\lambda_{t}^{d}$ goes up, which means the probability of default goes larger as well, it results that the LCDS Spread is more expensive. 
$<$ Figure 1 about here $>$

However, Figure 2 shows that the shape of LCDS term structures when we fix the common factor ${ }^{r_{t}}, X_{t}^{d}$, which is the specific factor of $\lambda_{t}^{d}$, the correlation parameter $\rho=-0.5$, and vary the mean $\theta^{p}$ and initial value $X_{0}^{p}$ for $X_{t}^{p}$ which is the specific factor of $\lambda_{t}^{p}$. We can see that the LCDS Spread of both figures decreases as $\theta^{p}$ or $X_{0}^{p}$ increases. Suppose the other parameters are fixed. If the initial value or mean of specific factor of $\lambda_{t}^{p}$ goes up, which means the probability of prepayment goes larger, it is an advantage for the investor of LCDS and results that the LCDS Spread is cheaper.

$<$ Figure 2 about here $>$

Figure 3 shows the impact of different coefficient ${ }^{\rho}$. The LCDS Spreads are greater with larger $|\rho|$, i.e. increasing the negative correlation between default and loan cancelation increases the LCDS Spread.

$<$ Figure 3 about here $>$

Figure 4 show the different path of $\lambda_{t}^{d}$ and $\lambda_{t}^{p}$. Since $2 \kappa \theta>\sigma^{2}, 2 \kappa^{i} \theta^{i}>\left(\sigma^{i}\right)^{2}, r_{t}$ and $X_{t}^{d}$ are non-negative process. And because we take $\eta$ is $1.5, \lambda_{t}^{d}$ will be a nonnegative process. We can see from the left picture that the path is all above zero. But, $\rho$ is negative so $\rho \eta r_{t}$ is non-positive process meanwhile $X_{t}^{p}$ is non-negative process, thus the sign of $\lambda_{t}^{p}$ is uncertain. If $\lambda_{t}^{p}>0$, it is at prepayment state, otherwise it is at inverse prepayment. We can see from the right picture that one of the three paths goes below zero at about year four and below zero since then. The other two paths are above zero.

$<$ Figure 4 about here $>$

Figure 5 show the impact of different $\eta$ when we fix the other parameters. $\eta$ represents the impact strength of common factor. If $\eta=0, \lambda_{t}^{d}=X_{t}^{d}, \lambda_{t}^{p}=X_{t}^{p}$ they are two independent process. The spread is lowest. We can see that the spread increase as $\eta$ increases. On the one hand, the increasing of $\eta$ increases the probability of default which increases the spread. On the other hand, since $\eta$ is negative, the increasing of $\eta$ will increase the probability that prepayment become negative which also increases the spread.

$<$ Figure 5 about here $>$

\section{Conclusion}

In this study, by using "top down" framework, we develop an intensity model for pricing a LCDS contract referenced a basket loan, where inverse prepayment is allowed. A closing-form solution under single-factor model and correlated CIR processes is obtained. This solution is easy to calculation, so that it is easy to apply to the practice. Some numerical calculation examples are presented, for which we get more direct view of the properties of the spread function and the relations among the parameters.

\section{References}

Dobranszky, P., (2008). Joint Modeling of CDS and LCDS Spreads with Correlated Default and Prepayment Intensities and with Stochastic Recovery Rate, Technical Report 08-04, Section of Statistics, K.U. Leuven.

Dobranszky, P., and Schoutens, W. (2008). Generic Levy One-Factor Models for the Joint Modeling of Prepayment and Default: Modeling LCDX, Technical Report 08-03, Section of Statistics, K.U. Leuven

Giesecke, K. (2008). Portfolio Credit Risk: Top Down vs. Bottom Up Approaches, Frontiers in Quantitative Finance: Credit Risk and Volatility Modeling

Hull, J., and White, A. (2000). Valuing Credit Default Swap I: No Counterparty Default Risk, Journal of Derivatives, 8(1) $29-40$.

Hull, J., and White, A. (2001). Valuing Credit Default Swap II: Modeling Default Correlation, Journal of Derivatives, $8(3) 12-22$.

Hull, J., and White, A. (2004). Valuation of a CDO and $n^{\text {th }}$ to Default CDS without Monte Carlo simulation, Journal of Derivatives, 12(2) 8-23. 
Jiang, LS. (2008). Mathematical Modeling and Cases Analysis of Financial Derivative Pricing, Higher Education Press Linetsky, V. (2004). Computing hitting time densities for CIR and OU diffusions: applications to mean-reverting models, J. Computational Finance, 7, No.4 1-22.

Shek, H., Uematsu S., and Zhen, W. (2007). Valuation of Loan CDS and CDX, working paper, Stanford University.

Zhen, W. (2007). Valuation of Loan CDS under Intensity Based Model, working paper, Stanford University.

Wu, S., Jiang, LS., and Liang, J. (2008). Pricing of Mortgage-Backed Securities with Repayment Risk, working paper.

Table1. Different parameter sets

\begin{tabular}{|c|c|c|}
\hline Figure & $\left(\kappa, \theta, \sigma, r_{0} ; \kappa^{d}, \theta^{d}, \sigma^{d}, X_{0}^{d} ; \kappa^{p}, \theta^{p}, \sigma^{p}, X_{0}^{p} ; \rho ; \eta\right)$ & Purpose \\
\hline 1(left) & $(0.2,0.3,0.2,0.2 ; 0.1, “ . ”, 0.1,0.1 ; 0.1,0.2,0.1,0.1 ;-0.5 ; 1)$ & Different $\theta^{d}$ \\
\hline 1(right) & $(0.2,0.3,0.2,0.2 ; 0.1,0.25,0.1, “ . ” ; 0.1,0.2,0.1,0.1 ;-0.5 ; 1)$ & Different $X_{0}^{d}$ \\
\hline 2(left) & $(0.2,0.3,0.2,0.2 ; 0.1,0.25,0.1,0.1 ; 0.1, “ ., 0.1,0.1 ;-0.5 ; 1)$ & Different $\theta^{p}$ \\
\hline 2(right) & $(0.2,0.3,0.2,0.2 ; 0.1,0.25,0.1,0.1 ; 0.1,0.2,0.1, “ . ” ;-0.5 ; 1)$ & Different $X_{0}^{p}$ \\
\hline 3 & $(0.2,0.3,0.2,0.2 ; 0.1,0.25,0.1,0.1 ; 0.1,0.2,0.1,0.1 ; “ . ” ; 1)$ & Different $\rho$ \\
\hline 4(left) & $(0.2,0.3,0.2,0.2 ; 0.1,0.25,0.1,0.1 ; 0.1,0.2,0.1,0.1 ;-0.5 ; 1.5)$ & Path of $\lambda_{t}^{d}$ \\
\hline 4(right) & $(0.2,0.3,0.2,0.2 ; 0.1,0.25,0.1,0.1 ; 0.1,0.2,0.1,0.1 ;-0.5 ; 1.5)$ & Path of $\lambda_{t}^{p}$ \\
\hline 5 & $(0.2,0.3,0.2,0.2 ; 0.1,0.25,0.1,0.1 ; 0.1,0.2,0.1,0.1 ;-0.5 ; “ . ”)$ & Different $\eta$ \\
\hline
\end{tabular}
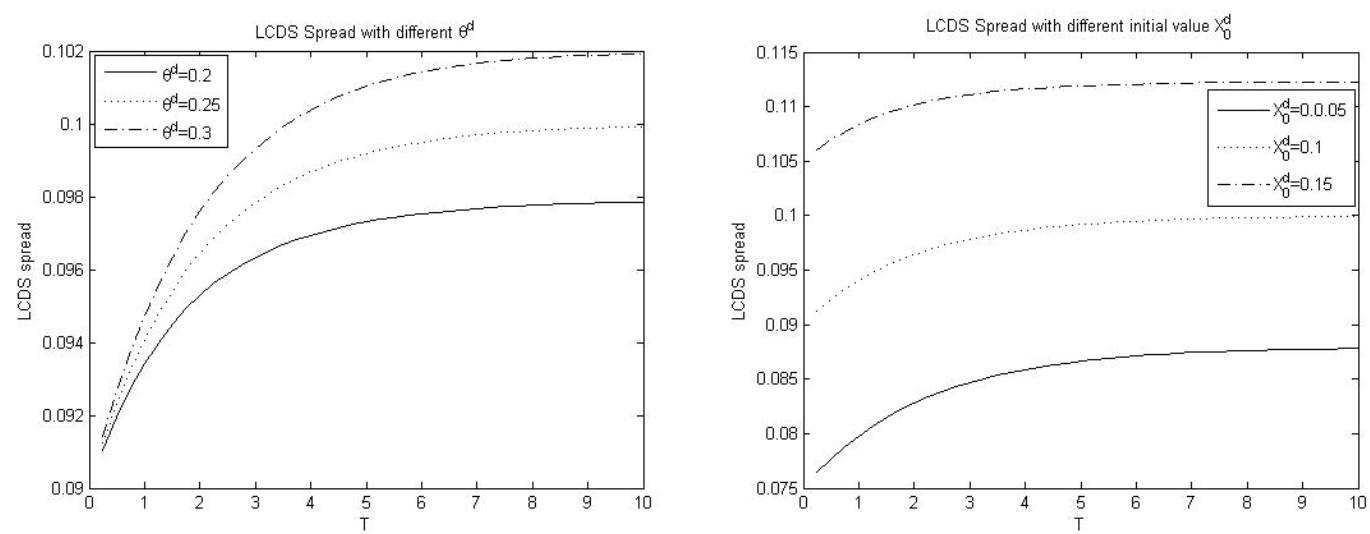

Figure 1. LCDS Spread vs. expired time $T$, varying $\theta^{d}$ (Left) and $X_{0}^{d}$ (Right)
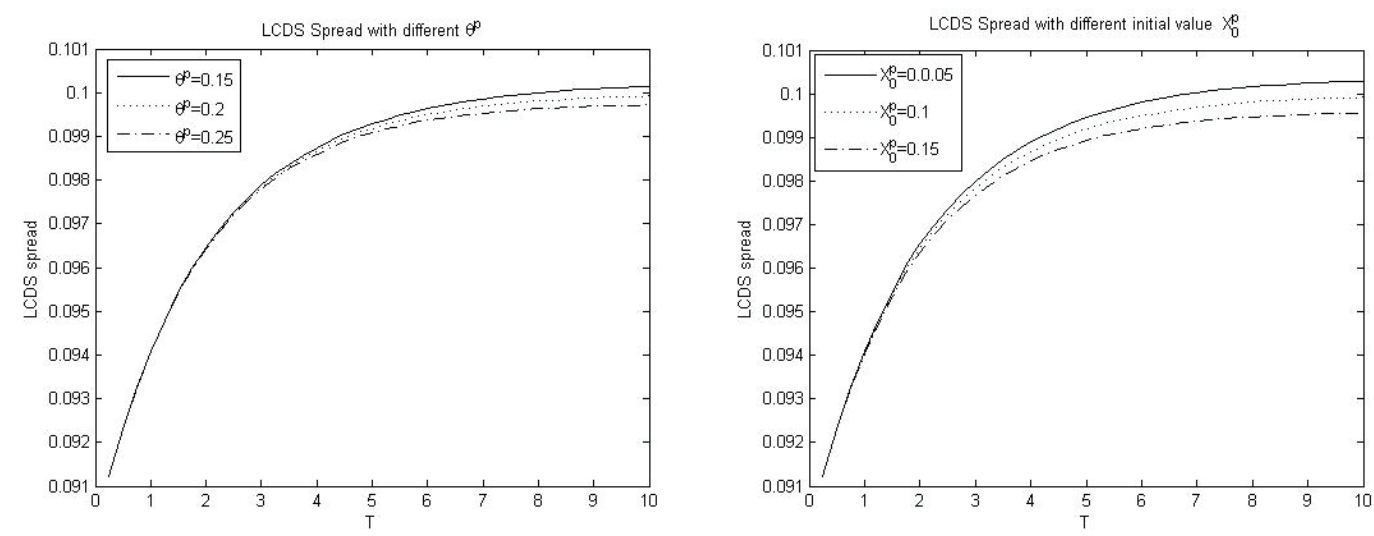

Figure 2. LCDS Spread vs. expired time $T$, varying $\theta^{p}$ (Left) and $X_{0}^{p}$ (Right) 


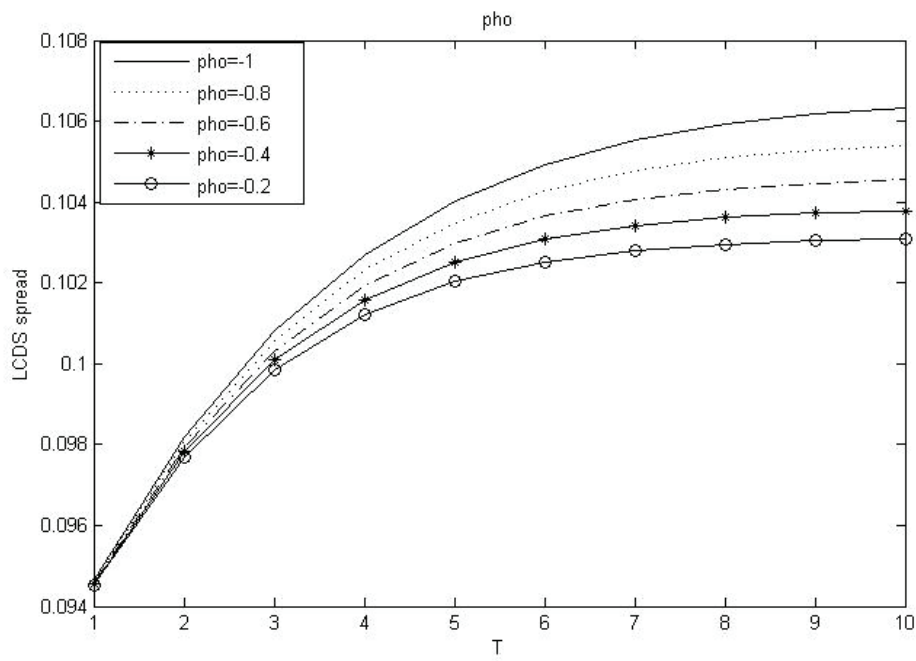

Figure 3. LCDS Spread vs. expired time $T$, varying $\rho$
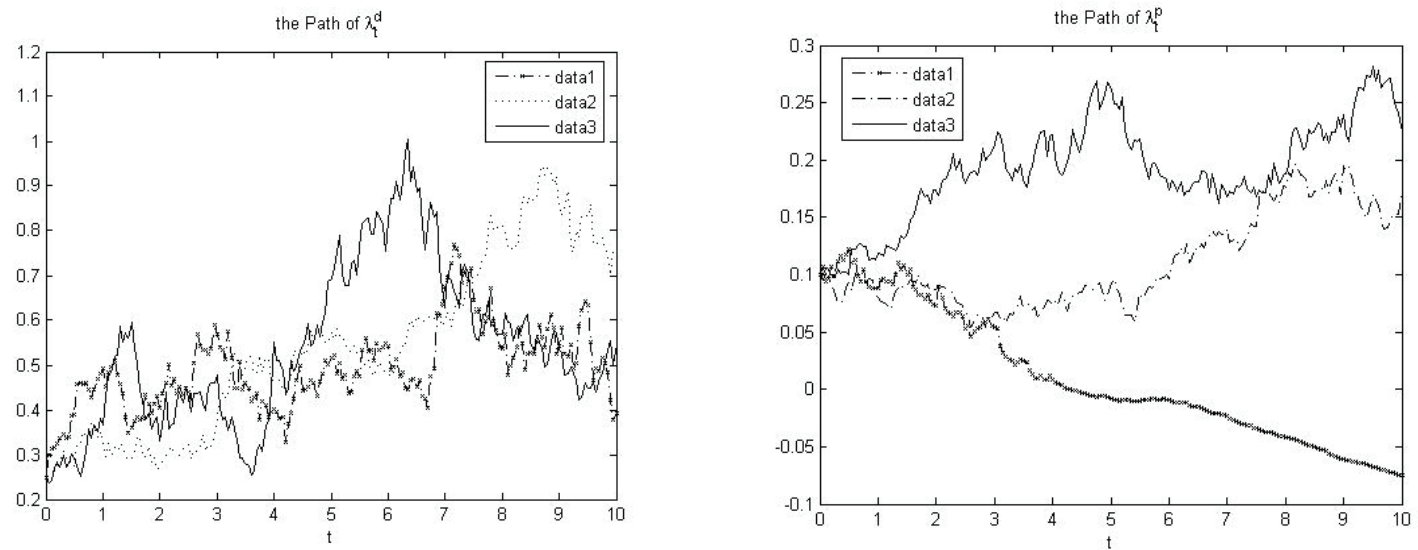

Figure 4. The different path of $\lambda_{t}^{d}$ (Left) and $\lambda_{t}^{p}$ (Right)
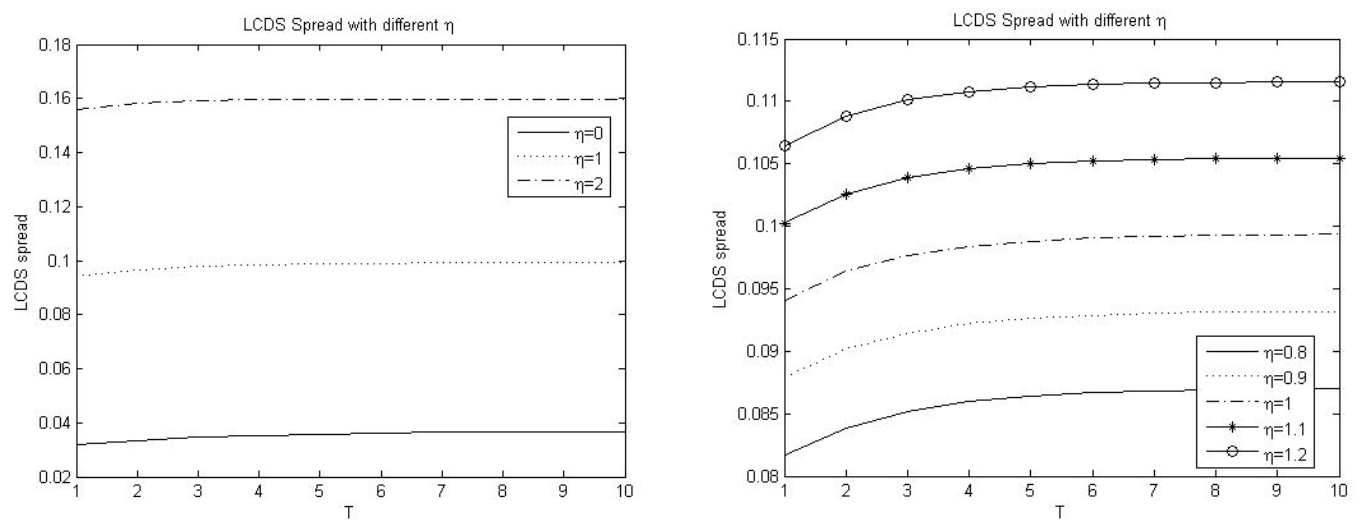

Figure 5. LCDS Spread vs. expired time $T$, varying $\eta$ 